\title{
The St. Jude Medical prosthesis
}

\section{A thirteen-year experience}

From May 1979 until July 1992, 1184 patients, 2 to 86 years of age (median = 59 years), received 1367 St. Jude Medical prostheses (578 aortic, 440 mitral, 3 tricuspid, and 170 multiple). Overall early mortality was $4 \%$ with $2.4 \%, 4.3 \%$, and $8.2 \%$ after aortic, mitral and multiple valve replacement, respectively. Follow-up was $100 \%$ complete (4936 patient-years). Actuarial survival at 10 years (including both early and late deaths) was $71 \% \pm 4.1 \%$ after aortic valve replacement, $\mathbf{5 8 . 8} \% \pm \mathbf{5 . 1} \%$ after mitral valve replacement, and $58.9 \% \pm \mathbf{9 . 6} \%$ after multiple valve replacement. Multivariate analysis identified age, previous operations, diabetes mellitus, extent of coronary artery disease, preoperative New York Heart Association class, and additional procedures as independent prognostic factors for overall survival. All patients including 20 children ( 2 to 18 years of age) received sodium warfarin. The linearized risk per 100 patient-years for all embolic events (major and minor) was $2.4,4.4$, and 5.0 after aortic, mitral, and multiple valve replacement, respectively; when only major events were considered, the linearized risks were $0.33,1.17,1.54$, respectively. Freedom from major systemic embolism at 10 years was $96 \% \pm 2 \%$ after aortic valve replacement, $88 \% \pm 4 \%$ after mitral valve replacement, and $89 \% \pm 3 \%$ after multiple valve replacement. Five patients had valve thrombosis, four of whom definitely received inadequate anticoagulation therapy. Ninety-seven patients had 107 episodes of anticoagulant-related hemorrhage (2.2/100 patient-years) accounting for 17 late deaths; 14 of the deaths involved an international normalized ratio in excess of 3.5. To reduce the rate of thromboembolism without increasing the rate of anticoagulant-related hemorrhage, we propose that the international normalized ratio should be kept between 2.5 and 3.0. No recorded structural failure or significant hemolysis was found in the absence of periprosthetic leak. This experience encourages us to continue using the St. Jude Medical prosthesis. (J Thorac CardiovasC SURG 1994;108:221-30)

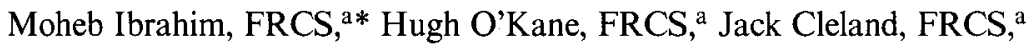
Dennis Gladstone, FRCS, ${ }^{\mathrm{a}}$ Mazin Sarsam, FRCS, ${ }^{\mathrm{a}}$ and Christopher Patterson, PhD, ${ }^{\mathrm{b}}$ Belfast, Northern Ireland

$\mathrm{W}_{\mathrm{it}}$ formance carefully. It is important that any observation

\footnotetext{
From the Department of Cardiac Surgery, Royal Victoria Hospital, ${ }^{a}$ and the Department of Epidemiology and Public Health, Queen's

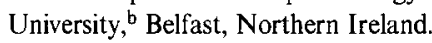

Received for publication Aug. 16, 1993.

Accepted for publication Mar, 16, 1994.

Address for reprints: Hugh O'Kane, FRCS, Department of Cardiac Surgery, Royal Victoria Hospital, Belfast, BT12 6BA, Northern Ireland.

*Present address: Division of Cardiothoracic Surgery, University of Ottawa Heart Institute, Ottawa Civic Hospital, 1053 Carling Ave., Ottawa, Ontario, Canada KIY 4E9.

Copyright ${ }^{(\odot)} 1994$ by Mosby-Year Book, Inc.

$0022-5223 / 94 \$ 3.00+0 \quad \mathbf{1 2} / \mathbf{1} / \mathbf{5 6 2 5 1}$
}

should be standardized, especially with regard to thromboembolic events, as suggested by McGoon, ${ }^{1}$ so that a reasonable attempt can be made to compare prostheses with each other. We introduced the St. Jude Medical prosthesis into our unit in 1979 and herein record a 13-year follow-up presented strictly within the guidelines proposed by the Society of Thoracic Surgeons. ${ }^{2}$

\section{Patients and methods}

Follow-up data were obtained during a 4-month closing interval (August 1992 until the end of November 1992). Between May 1979 and July 1992, 1184 patients received 1367 St. Jude Medical prostheses (St. Jude Medical, Inc., St. Paul, Minn.); 515 were male (43.5\%) and 669 were female (56.5\%) patients, ranging from 2 to 86 years of age (median $=59$ years), with $11 \%$ being 70 years of age or older. Four hundred ten patients $(35 \%)$ had previous cardiac operations. In the preoper- 
Table I. Early mortality in the different subgroups

\begin{tabular}{lrc}
\hline & No. of patients & Early deaths \\
\hline AVR & & \\
$\quad$ Isolated & 430 & $10(2 \%)$ \\
Plus CABG & 97 & $1(1 \%)$ \\
$\quad$ Plus procedures other than CABG & 49 & $3(6 \%)$ \\
$\quad$ Total & 576 & $14(2.4 \%)$ \\
MVR & & \\
$\quad$ Isolated & 334 & $9(3 \%)$ \\
Plus CABG & 45 & $4(9 \%)$ \\
$\quad$ Plus procedures other than CABG & 59 & $6(10 \%)$ \\
$\quad$ Total & 438 & $19(4.3 \%)$ \\
Multiple valve replacement & & \\
$\quad$ Isolated & 138 & $7(5 \%)$ \\
$\quad$ Plus CABG & 11 & $1(10 \%)$ \\
$\quad$ Plus procedures other than CABG & 21 & $6(29 \%)$ \\
$\quad$ Total & 170 & $14(8.2 \%)$ \\
Total & 1184 & $47(4 \%)$ \\
\end{tabular}

ative period, $56 \%$ of patients were in New York Heart Association (NYHA) functional class III, 26\% were in class IV, and the remainder were in class I or II. Of the valves that were implanted one at a time, 578 were aortic, 440 were mitral, and three were tricuspid. One hundred fifty-eight patients underwent aortic and mitral valve replacement (AVR + MVR), five underwent mitral and tricuspid valve replacement (MVR + TVR), one underwent aortic and tricuspid valve replacement (AVR + TVR), and six underwent triple valve replacement (AVR + MVR + TVR). In 284 cases (24\%), additional procedures were required. Coronary artery bypass grafting (CAGB) was performed in 13\% (97 AVR, 45 MVR, 11 double valve), 83 patients had other valves repaired, 11 had correction of a congenital defect, 10 had replacement of the ascending aorta, 9 had resection of subaortic stenosis, and the remaining 18 had miscellaneous procedures.

Operative technique included cardiopulmonary bypass with moderate hypothermia $\left(28^{\circ}\right.$ to $\left.32^{\circ} \mathrm{C}\right)$, topical cooling, and either crystalloid or blood cardioplegic solution. A continuous suture technique with polypropylene was used to insert 1339 valves, an interrupted suture technique was used for 20 valve replacements ( 11 mitral and 9 aortic), and a mixed technique was used for eight MVRs. Each valve was soaked in a 1\% neomycin solution before insertion.

Sodium warfarin therapy was started either 48 hours after the operation or as soon as the chest drains were removed; the aim was to achieve an international normalized ratio of 2.0 to 2.5 (prothrombin time ratio of 1.7 to 1.9.) One surgeon administered subcutaneous heparin (5000 IU thrice daily) simultaneously until this international normalized ratio range was achieved. Two surgeons used dipyridamole ( $100 \mathrm{mg}$ daily) in addition.

All patients who were still alive during the closing interval were contacted by telephone or through their family doctors. In addition, hospital records were reviewed, and the cardiologists in charge of the patients were contacted as necessary. Clinical details on deceased patients were obtained from hospital records, family doctors, the Office of the Registrar General, and relatives. In $26 \%$ of the deceased patients, postmortem examination was carried out. Echocardiographic studies to exclude periprosthetic leaks were performed as necessary based on clin- ical indications. Follow-up was $100 \%$ complete with a total of 4936 patient-years (aortic 2441 patient-years, mitral 1782 patient-years, and multiple 713 patient-years). The mean follow-up was 4.2 years.

This report strictly follows the guidelines of the Society of Thoracic Surgeons ${ }^{2}$ for reporting morbidity and mortality after cardiac valvular operations. It is worth emphasizing that the term periprosthetic leak is used to indicate leaks in the absence of infection. In addition, McGoon's' strictures concerning the reporting of embolic events were adhered to. Patients were specifically asked if they had experienced blurred or double vision, dizziness, numbness, weakness, inability to move a part or to speak, blackout, or a stroke. Dizziness was considered relevant if it occurred repeatedly and suddenly, in the absence of sudden movements or change of position, and if the patient had no ear problems. Dizzy spells occurring both before and after the operation were not included under the category of embolic events.

Statistical methods. Early mortality rates were compared in subgroups of the patients defined by potential prognostic variables. Univariate comparisons were performed with the $\chi^{2}$ test (or Fisher's exact probability test). Logistic regression analysis was then used to define a subset of variables that were independently predictive of early mortality. Only variables that gave significant likelihood ratio statistics were retained in the model. Results were summarized as relative odds on early death with 95\% confidence limits.

The actuarial method was used to summarize late mortality and overall mortality. Survival estimates are reported \pm standard error of the estimate. Univariate comparisons were performed with Breslow's test. Cox's proportional hazards model was then used to identify independent prognostic variables. Partial-likelihood ratio tests were used, and only significant variables were retained in the model. Results were summarized as relative hazards with $95 \%$ confidence limits. Tests were conducted at the $5 \%$ level of significance. All analyses were performed with the SPSS for Windows software package (SPSS Inc., Chicago, Ill.).

\section{Results}

Early mortality. Forty seven patients (4\%) died within 30 days or during the same hospital admission even if the hospital stay was longer than 30 days. Sixty percent of these cases had a postmortem examination carried out. Mortality according to the location of the prostheses was $2.4 \%$ after AVR, $4.3 \%$ after MVR, and $8.2 \%$ after multiple valve replacement (Table I). Thirteen patients died on the operating table, including three who had a ruptured left ventricle during MVR. The other causes of early mortality were multiple organ failure $(n=13)$, congestive heart failure $(n=5)$, arrhythmia $(n=4)$, tamponade $(n=2)$, intraoperative cerebrovascular accidents $(n=3)$, postoperative cerebral infarcts $(n=3)$, further aortic dissection $(n=1)$, prosthetic endocarditis $(n=1)$, and septicemia $(n=2)$.

Univariate analysis identified the following four risk factors for early mortality: previous operation, additional procedures other than CABG, NYHA functional class 
Table II. Risk factors for early mortality identified by logistic regression analysis

\begin{tabular}{lcc}
\hline \multicolumn{1}{c}{ Covariate } & $\begin{array}{c}\text { Relative odds } \\
(95 \% \text { CL) }\end{array}$ & $\begin{array}{c}\mathrm{p} \mathrm{Value} \\
\text { (overall) }\end{array}$ \\
\hline $\begin{array}{c}\text { Site of implantation } \\
\text { MVR versus AVR }\end{array}$ & 0.04 \\
$\quad \begin{array}{l}\text { Multiple versus AVR } \\
\text { Additional procedures }\end{array}$ & $2.85(1.29,6.29)$ & \\
$\quad$ CABG versus none & $1.60(0.64,4.01)$ & 0.0006 \\
$\quad \begin{array}{l}\text { Other versus none } \\
\text { Preoperative NYHA class }\end{array}$ & $4.14(2.11,8.11)$ & \\
$\quad$ Class III and IV versus I and II & $3.62(1.06,12.4)$ & 0.04 \\
\hline
\end{tabular}

CL, Confidence limits.

III or IV, and multiple valve replacement compared with AVR. The extent of coronary artery disease, CABG, age, gender, and diabetes mellitus were not significantly associated with early mortality on univariate analysis. Although early mortality in the multiple valve replacement group was higher than that in the MVR group ( $8.2 \%$ and $4.3 \%$, respectively), the difference was not statistically significant. Logistic regression analysis of early mortality showed that only three variables were independently predictive of early death: site of implantation, additional procedures, and preoperative NYHA functional class (Table II).

When the analysis of early mortality was conducted in subgroups defined by location of prosthesis, aortic regurgitation compared with stenosis was a significant risk factor on both univariate analysis and logistic regression analysis. The nature of the mitral lesion (regurgitation versus stenosis) was not associated with increased risk.

Late mortality. One hundred sixty patients $(14 \%)$ had died by the time of the closing interval, $26 \%$ of whom had postmortem examination. The causes of late death are listed (Tables III and IV). Diagnosis was deferred in two patients because we were unable to ascertain the exact cause of death. The sudden unexplained death in the AVR case without an autopsy was regarded as valve related (Table IV). No recorded deaths were due to structural failure.

The risk factors for late survival identified on univariate survival analysis were as follows: previous operations, diabetes mellitus, extent of coronary disease, preoperative NYHA functional class III and IV, previous operation, and MVR compared with AVR. The comparison between MVR and multiple valve replacement was not significant. Gender and additional procedures including CABG were not significantly associated with late mortality.

The Cox's model analysis indicated that age group, previous operation, diabetes mellitus, extent of coronary
Table III. Cardiac non-valve-related and noncardiac causes of late mortality

\begin{tabular}{lc}
\hline & $\begin{array}{c}\text { No. of } \\
\text { patients }\end{array}$ \\
\hline Cardiac non-valve-related & \\
Congestive heart failure & 39 \\
Myocardial infarction & 38 \\
Arrhythmia & 9 \\
Dissection of the ascending aorta & 2 \\
Total & 88 \\
Noncardiac & \\
Malignancy & 24 \\
Bronchopneumonia & 4 \\
Hemorrhagic pancreatitis & 1 \\
Ruptured abdominal aortic aneurysm & 1 \\
AIDS-related infection & 1 \\
Hypoglycemic coma & 1 \\
Acute pyelonephritis & 1 \\
Jacob-Creutzfeldt's disease & 1 \\
Septicemia & 1 \\
Pneumococcal meningitis & 1 \\
Total & 36 \\
\hline
\end{tabular}

AIDS, Acquired immunodeficiency syndrome.

Table IV. Causes of valve-related late mortality and location of prosthesis

\begin{tabular}{lccc}
\hline \multicolumn{1}{c}{ Cause of death } & AVR & MVR & Multiple \\
\hline Cerebral hemorrhage & 5 & 7 & 2 \\
Subarachnoid hemorrhage & 0 & 1 & 0 \\
Gastrointestinal hemorrhage & 1 & 1 & 0 \\
Mesenteric infarction & 0 & 0 & 2 \\
Cerebral infarct & 1 & 2 & 1 \\
Valve thrombosis & 0 & 0 & 1 \\
Prosthetic valve endocarditis & 2 & 4 & 0 \\
Periprosthetic leak & 0 & 2 & 1 \\
Sudden death & 1 & 0 & 0 \\
Total & 10 & 17 & 7
\end{tabular}

artery disease, and additional procedures were independently prognostic of late mortality (Table $\mathrm{V}$ ).

When the analysis of late mortality was conducted in subgroups defined by location of prostheses, aortic regurgitation compared with aortic stenosis was found to be a risk factor only after univariate analysis. The nature of the mitral valve lesion (regurgitation versus stenosis) was not associated with increased risk.

Overall survival. The overall survival (including both early and late mortality) is shown in Fig. 1. Of the 977 patients who were alive by the time of the study, $88 \%$ were in NYHA functional class I or II, and $12 \%$ were in class III or IV.

Risk factors associated with overall mortality (early and late) were first identified with univariate analysis. The risk factors so identified were age, previous operation, 
Table V. Prognostic factors for late mortality identified by Cox's model

\begin{tabular}{lcc}
\hline \multicolumn{1}{c}{ Covariate } & $\begin{array}{c}\text { Relative hazard } \\
(95 \% \mathrm{CL})\end{array}$ & $\begin{array}{c}\mathrm{p} \text { Value } \\
\text { (overall) }\end{array}$ \\
\hline Age group $(\mathrm{yr})$ & $16.0(2.22,114)$. & \\
40-69 versus $<40$ & $25.3(3.33,192)$. & \\
$\geq 70$ versus $<40$ & $2.07(1.50,2.86)$ & $<0.0001$ \\
Previous operation & $2.23(1.38,3.59)$ & 0.003 \\
Diabetes mellitus & & $<0.0001$ \\
Extent of coronary artery disease & $3.18(1.85,5.45)$ & \\
$\quad$ Single versus none & $5.91(3.43,10.2)$ & \\
$\quad$ Double/triple versus none & & 0.002 \\
Additional procedures & $0.33(0.18,0.61)$ & \\
$\quad$ CABG versus none & $1.00(0.61,1.65)$ & \\
Other versus none & & \\
\hline
\end{tabular}

$C L$, Confidence limits.

diabetes, extent of coronary disease, preoperative NYHA functional class III and IV, and additional procedures. Cox's model analysis indicated that age group, previous operation, diabetes mellitus, extent of coronary artery disease, preoperative NYHA class, and additional procedures were independently prognostic of overall survival (Table VI).

When the analysis of overall survival was conducted in subgroups of patients, defined by location of prosthesis, aortic regurgitation and mixed aortic lesion compared with stenosis were identified as risk factors with univariate analysis. Cox's model analysis gave estimates of relative hazard of 2.9 (95\% confidence limits: $1.6,5.2)$ and 1.9 (95\% confidence limits: $1.0,3.4$ ), respectively, for these two groups (i.e., aortic regurgitation and mixed aortic lesion) compared with the aortic stenosis group after adjustment for the other risk factors.

Systemic embolism. Ninety-eight patients $(8.3 \%)$ had 174 embolic events (40 major and 134 minor). ${ }^{*}$ Nine of the events were fatal, seven cerebral and two mesenteric. Sixty-eight patients had only one embolic event, eleven had two events, eleven had three events, and eight had more than three events. The great majority $(89 \%)$ of embolic events were cerebral. In 41 patients, the international normalized ratio at the time of the event was within our accepted therapeutic range ( 2.0 to 2.5 ), whereas in 62 events it was below the therapeutic range $(<2.0)$. The international normalized ratio at the time of the event was not available in 71 episodes, the majority of which (66 of 71) were minor and the patient therefore was not admitted to the hospital.

The freedom from all embolic events is shown in Fig.

*:"Minor representing a transient episode and major indicating permanent residual effect or the requirement for operation or other significant therapeutic modality ...."
Table VI. Prognostic factors for overall survival identified by Cox's model

\begin{tabular}{lcc}
\hline & $\begin{array}{c}\text { Relative hazard } \\
(95 \% \text { CL) }\end{array}$ & $\begin{array}{c}\mathrm{p} \mathrm{Value} \\
\text { (overall) }\end{array}$ \\
\hline Age group (yr) & & 0.0001 \\
$\quad 40-69$ versus $<40$ & $5.92(1.88,18.7)$ & \\
$\quad \geq 70$ versus $<40$ & $8.23(2.42,28.0)$ & \\
Previous operation & $1.91(1.43,2.55)$ & $<0.0001$ \\
Diabetes mellitus & $1.83(1.17,2.87)$ & 0.015 \\
Extent of CAD & & $<0.0001$ \\
$\quad$ Single versus none & $2.53(1.53,4.18)$ & \\
$\quad$ Double/triple versus none & $5.01(3.02,8.33)$ & \\
Preoperative NYHA class & & 0.04 \\
$\quad$ Class III and IV vs I and II & $1.52(1.01,2.30)$ & \\
Additional procedures & & 0.0004 \\
$\quad$ CABG versus none & $0.40(0.23,0.70)$ & \\
$\quad$ Other versus none & $1.47(1.01,2.16)$ & \\
\hline CL, Confidence limits. & &
\end{tabular}

2 and was significantly better after AVR than after either MVR or multiple valve replacement. No significant difference was found between the MVR group and the multiple valve replacement group.

The linearized risk, taking both major and minor episodes into account, was 3.5/100 patient-years for all patients, 2.4/100 patient-years for AVR, 4.4/100 patient-years for MVR, and 5.0/100 patient-years for multiple valve replacement. When only major episodes were considered, the linearized risk was $0.3,1.2$, and 1.5 / 100 patient-years after AVR, MVR, and multiple valve replacement, respectively (an overall linearized risk of $0.8 / 100$ patient-years). Although the rate of systemic embolism was higher in patients in atrial fibrillation (10.3\%) compared with those in sinus rhythm (7.1\%), the difference was not statistically significant. Estimates for freedom from major embolic events are shown in Fig. 3.

Valve thrombosis. Valve thrombosis occurred in five patients, two in the AVR group, two in the MVR group, and one in the AVR + MVR group. In the latter case, diagnosis was made at autopsy, which showed a large thrombus on the mitral prosthesis and a smaller one on the aortic prosthesis. This patient had been in atrial fibrillation, and the international normalized ratio on admission was within the therapeutic range. In the other four patients, the international normalized ratio was subtherapeutic, the patients had their thrombosed prostheses replaced, and they were all alive at the time this article was written.

Anticoagulant-related hemorrhage. Ninety-seven patients (8.2\%) had 107 episodes of hemorrhage necessitating hospital admission or blood transfusion or resulting in death. Ten patients each had two hemorrhagic episodes, and two of these died of intracranial hemorrhage. 


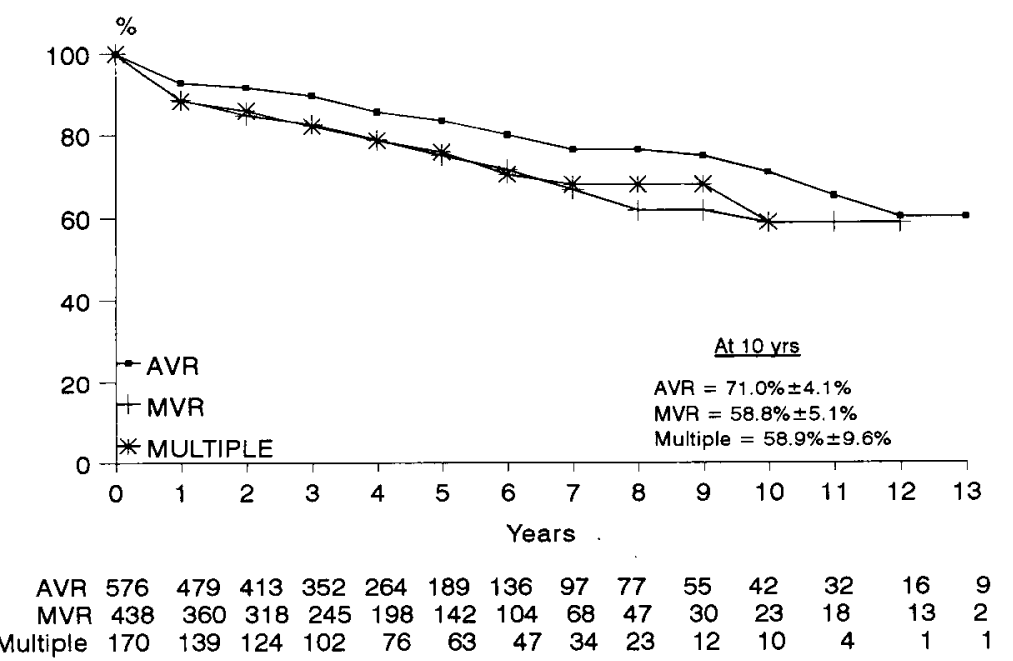

Fig. 1. Actuarial survival of all patients, including hospital and 30-day mortality after AVR, MVR, and multiple valve replacement.

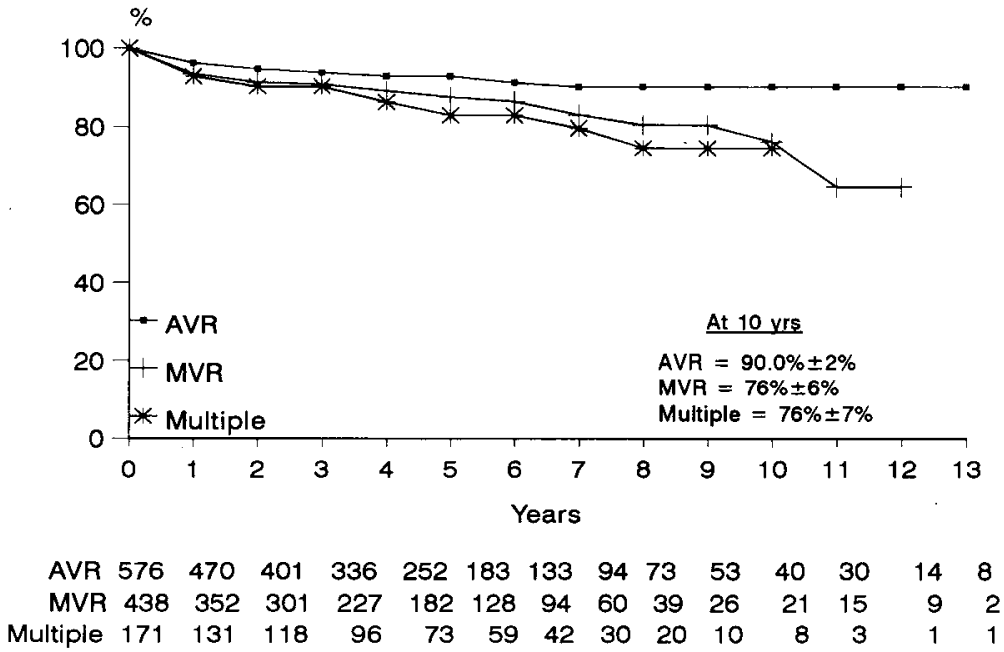

Fig. 2. Freedom from all embolic events (major and minor) after AVR, MVR, and multiple valve replacement.

In 101 episodes $(94 \%)$, the international normalized ratio at the time of the episode was known. In 82 cases, it was above our therapeutic range, whereas in 19 it was within our range. Seventeen episodes were fatal. In 14 of these cases, the international normalized ratio was known at the time of the hemorrhage and it was found to be in excess of 3.5. The vast majority of these fatalities (15 of 17) were due to intracranial hemorrhage, with the remaining two deaths resulting from severe gastrointestinal bleeding. An additional eight patients had nine episodes of subdural hematoma, seven requiring emergency burr hole drainage. The linearized risk of anticoagulant-related hemorrhage was $2.2 / 100$ patient-years for all patients, $1.7 / 100$ patient-years for AVR, 2.8/100 patient-years for MVR, and 2.1/100 patient-years for multiple valve replacement.

Estimates for freedom from anticoagulant-related hemorrhage are shown in Fig. 4. The risk of anticoagulant-related hemorrhage was significantly greater with MVR than with AVR ( $p=0.004)$. However, no significant difference was found between the AVR and multiple valve replacement groups.

Prosthetic valve endocarditis. Thirteen patients had 14 episodes of prosthetic valve endocarditis; six occurred within 3 months (early) and eight occurred late. Of these thirteen patients seven died, and none of the seven had preoperative endocarditis. Of the six patients who had early prosthetic valve endocarditis, only one had bacterial endocarditis before the initial valve replacement. 


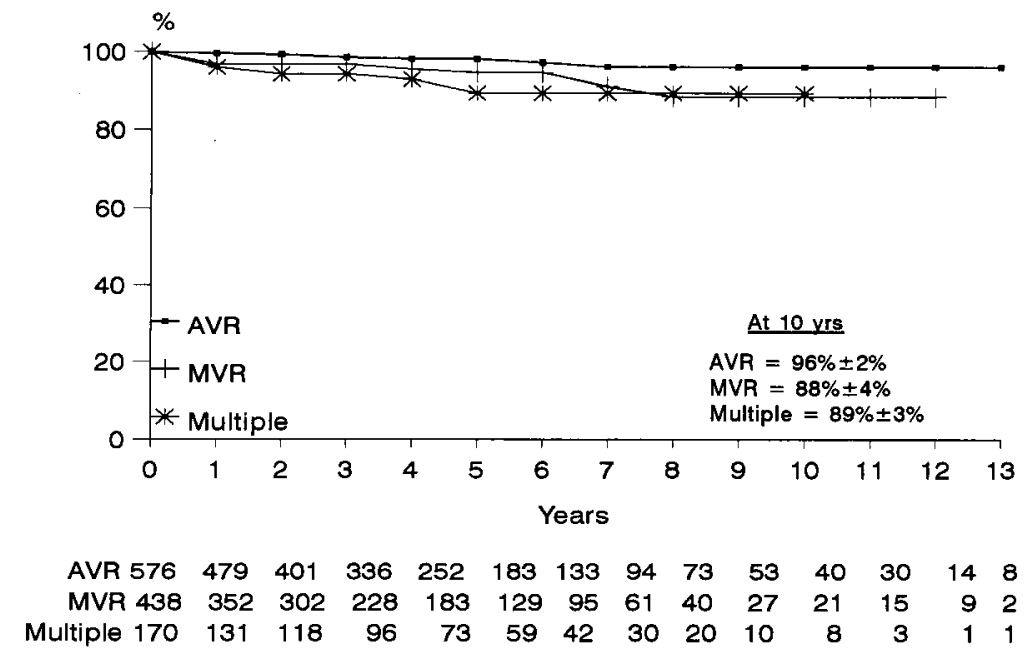

Fig. 3. Freedom from major embolic events after AVR, MVR, and multiple valve replacement.

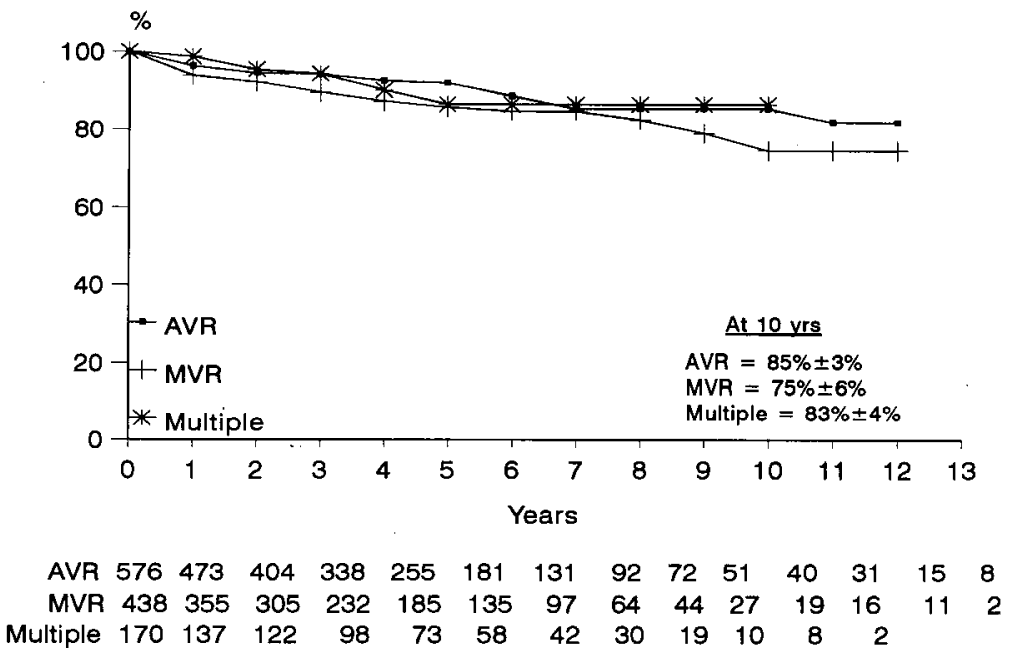

Fig. 4. Freedom from anticoagulant-related hemorrhage after AVR, MVR, and multiple valve replacement.

Among forty patients who had preoperative bacterial endocarditis, only one had postoperative prosthetic valve endocarditis (early), and the responsible organism (Candida) was different from the preoperative organism. The rate of early prosthetic valve endocarditis was $2.5 \%$ ( 1 of 40) among patients with preoperative bacterial endocarditis compared with $0.44 \%$ (5 of 1144) among those without infection, this increase was not statistically significant (Fisher exact probability test, $p=0.19$ ). The linearized risk per 100 patient-years of prosthetic valve endocarditis (early and late) was 0.3 for all patients, 0.1 for AVR, 0.2 for MVR, and 1.0 for multiple valve replacement.

Periprosthetic leak. Forty-three patients had 44 periprosthetic leaks and necessitated 14 reoperations in 13 patients, 13 in the mitral and one in the aortic area $(p<0.001)$. The linearized risk per 100 patient-years was 0.87 for all patients, 0.3 after AVR, 1.5 after MVR, and 1.26 after multiple valve replacement. The linearized risk per 100 patient-years for periprosthetic leak necessitating reoperation was 0.28 for all patients. The actuarial freedom from periprosthetic leak is shown in Fig. 5.

Reoperation. Twenty-one reoperations were performed in 20 patients: four AVR, $12 \mathrm{MVR}$, and four with multiple valve replacement. Indications for reoperation included periprosthetic leak in 14 patients (four of whom died), prosthetic valve endocarditis in two patients (one of whom died), and valve thrombosis in four patients (none 


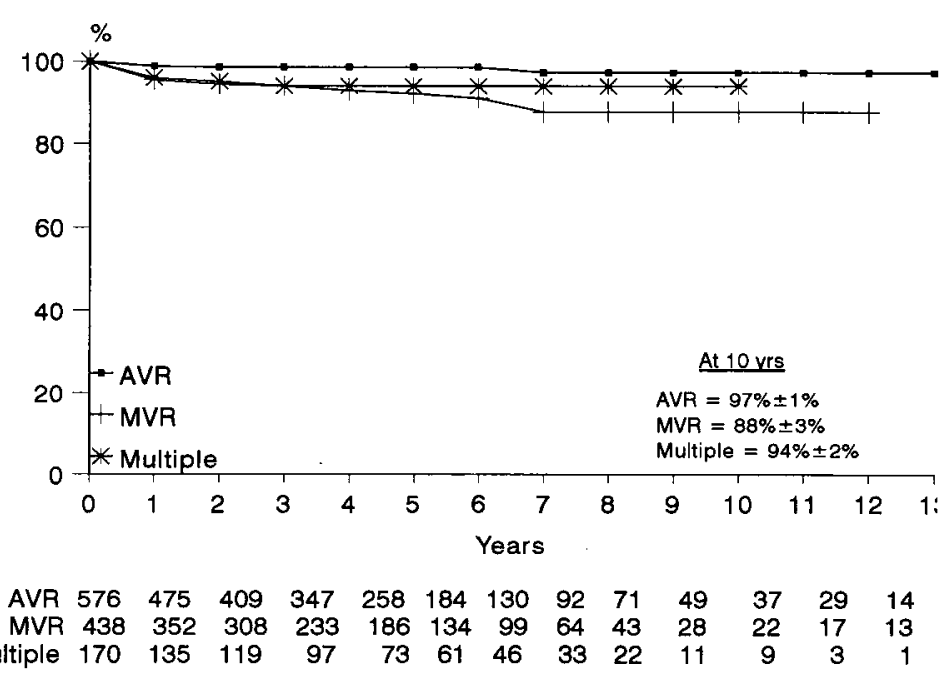

Fig. 5. Freedom from periprosthetic leak after AVR, MVR, and multiple valve replacement.

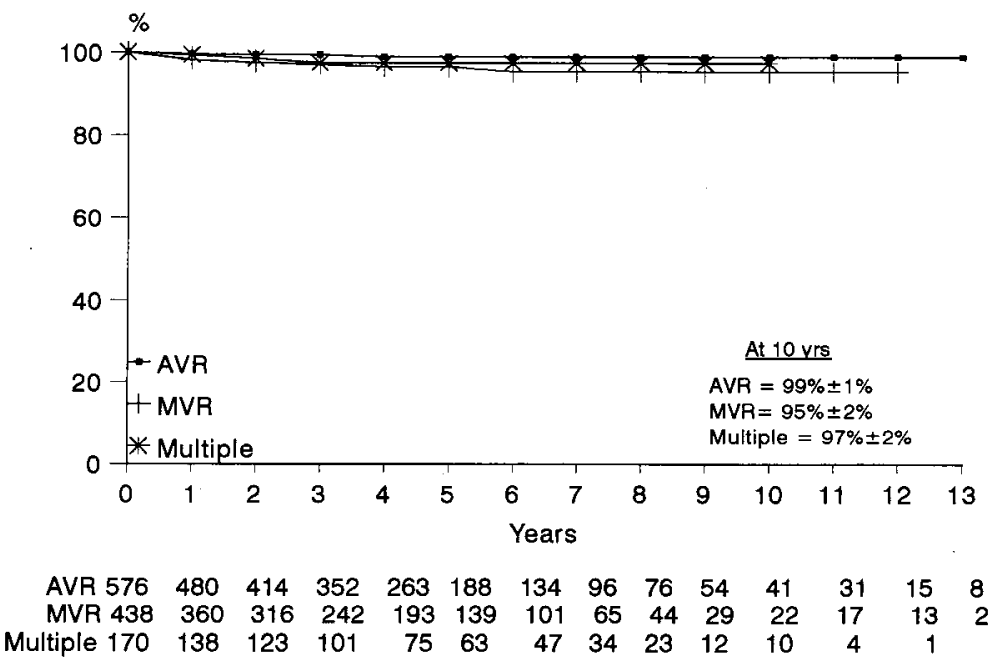

Fig. 6. Freedom from reoperation after AVR, MVR, and multiple valve replacement.

of whom died). All of the foregoing deaths occurred in the perioperative period. The linearized risk for reoperation per 100 patient-years was 0.42 for all patients, 0.16 for AVR, 0.67 for MVR, and 0.7 for multiple valve replacement. The actuarial estimate of the percentage free from reoperation is shown in Fig. 6.

Prosthetic valve hemolysis. No valve required replacement for hemolysis in the absence of a periprosthetic leak.

Freedom from all valve-related events. Embolic complications, valve thrombosis, anticoagulant-related hemorrhage, prosthetic valve endocarditis, periprosthetic leak, reoperation, and valve-related death were reviewed overall for AVR, MVR, and multiple valve replacement. The actuarial estimate of the percentage free from a valve-related event is shown in Fig. 7. A significant difference was found in the occurrence of valve-related events between the AVR group and each of the other groups.

\section{Discussion}

Our results once again draw attention to the fact that thromboembolism and anticoagulant-related hemorrhage remain the most numerous and life-threatening complications of mechanical prosthetic valves. They were the major causes of our valve-related late mortality, accounting for 24 deaths ( $71 \%$ ). In the 14 hemorrhagerelated deaths in which the international normalized ratio was known, the ratio was above 3.5 at the time of the event. This finding would lead us to hypothesize that if the 


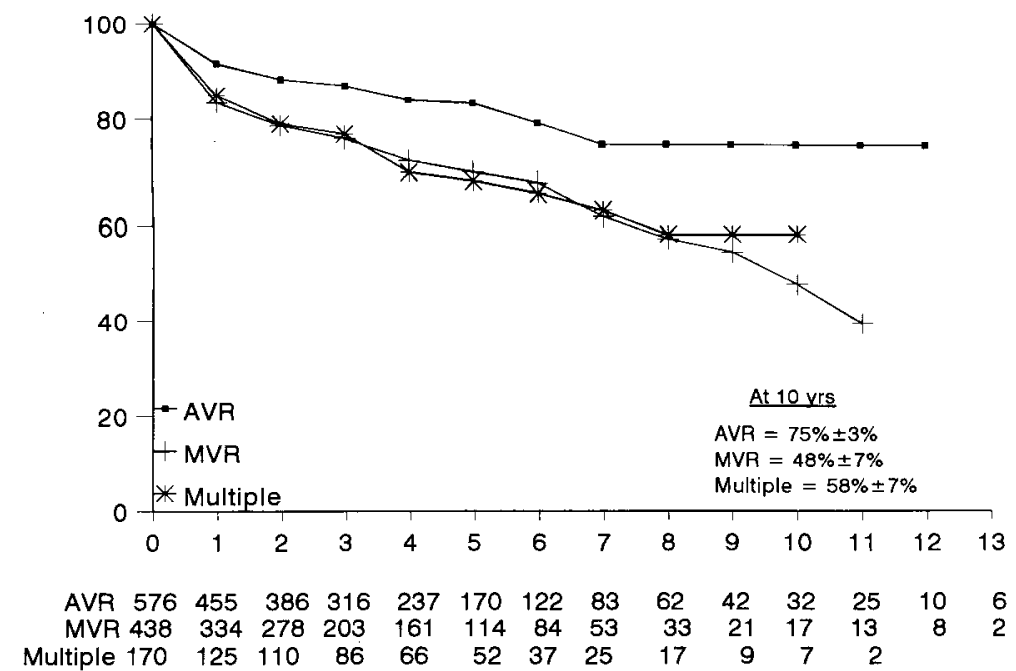

Fig. 7. Freedom from all valve-related morbidity and mortality after AVR, MVR, and multiple valve replacement.

international normalized ratio was not allowed to exceed an upper limit of 3.0 the prevalence of anticoagulant-related hemorrhage would fall. At same time we must be aware of what could happen to the embolic events if we so adjusted the international normalized ratio. From our own results we know that the international normalized ratio was under 2.5 in all 103 embolic events in which it was known, which included 35 of the major events $(88 \%)$. Combining these two observations, we postulate that an international normalized ratio in the range of 2.5 to 3.0 would be the optimum for reducing the amount of both embolic events and anticoagulant-related hemorrhage.

Our conclusion is in accordance with that of Horskotte and associates ${ }^{3}$ who state that an international normalized ratio of 2.5 to 3.0 would reduce thromboembolic and bleeding events in patients with St. Jude Medical prostheses. These authors recommend a higher international normalized ratio (2.7 to 3.6) for patients with mitral St. Jude Medical prostheses who are in atrial fibrillation, but we would be reluctant to recommend an international normalized ratio above 3.0 because of our fear of fatal hemorrhage. In addition, although we found the rate of systemic embolism was higher in patients in atrial fibrillation (10.3\%) compared with those in sinus rhythm $(7.1 \%)$, the difference was not significant.

When we tried to do a comparative analysis of our prevalence of anticoagulant-related hemorrhage and thromboembolic complications with those in the literature, we were frustrated by a lack of conformity in the reported results. In none of the reports we looked at ${ }^{4-14}$ could we find a record of the state of anticoagulation at the time of a hemorrhagic episode. Similarly for thromboembolism, no standard form of reporting events was found. Some reported only major emboli, ${ }^{7}$ others reported events only after the first 6 months, ${ }^{15}$ and with others ${ }^{4,5}$ it was not clear how many patients had recurrent episodes or whether each event was included in their calculation of the linearized risks. Where we could find reasonably comparable definitions of thromboembo$\operatorname{lism}^{12,13}$, our overall embolic rate was similar. With those authors ${ }^{7}$ who only recorded their major events, we found that we had a much lower prevalence of major events $(0.3$ versus 2.1 for AVR, 1.2 versus 4.3 for MVR, and 1.5 versus 4.6 for multiple valve replacement). This experience reinforces the difficulty in comparing the results from different groups that do not adhere to a standard definition. The excellent paper written by $\mathrm{McGoon}^{1}$ in 1984 gives guidelines for reporting thromboembolic complications which could be followed before papers are submitted and accepted for publication. The adherence to these guidelines and the guidelines approved by the Council of the Society of Thoracic Surgeons ${ }^{2}$ would remove some of the difficulty in comparing the results of different valve prostheses.

Prosthetic valve endocarditis was the next major cause of morbidity and mortality (after thromboembolism and anticoagulant-related hemorrhage). Our linearized prevalence of prosthetic valve endocarditis is similar to that of other authors ${ }^{4,5,7,11}$ reporting experience with different prostheses but is lower than that reported by Lund and associates ${ }^{14}$ with the Starr-Edwards prosthesis (Baxter Healthcare Corp., Edwards Division, Santa Ana, Calif.). Six of the seven patients who died had their prosthetic valve endocarditis late (i.e., after 3 months). Therefore, the reduction of prosthetic valve endocarditis depends not only on a rigid aseptic technique at the time of operation 
but just as importantly on the careful advice to patients concerning possible sources of bacteremia and their prompt treatment.

The analysis of the effect that CABG has on early and late mortality gave us interesting results. For early mortality, CABG was associated with a slight but not statistically significant increase in risk (Table II), whereas for late mortality, CABG was identified as a factor that positively affected survival (Table VI) when the extent of the patient's coronary artery disease is taken into account. The beneficial effect on late mortality is most likely due to a decrease in ischemic events and an improvement in left ventricular function over the years. Our overall survival results would encourage us to perform CABG in addition to valve replacement for its protective long-term effect, even though we found it to be associated with a slight increase in the risk of early mortality.

Surprisingly, we found that older age was neither a univariate nor a multivariate predictor of early mortality in our series. This removal of age as a risk factor is probably related to the energetic and detailed attention we pay to the immediate postoperative care of the elderly, especially regarding renal and respiratory problems. This experience, however, is not shared by others ${ }^{16}$ who have identified age as an important risk factor both for MVR and AVR. Age still remained a negative prognostic factor for late mortality (Table $\mathrm{V}$ ) and for overall survival (Table VI).

The increased early mortality that we found after MRV and multiple valve replacement could not be accounted for by either the NYHA preoperative class or the higher rate of previous operation in these cases, and the results of our analysis led us to conclude that this increased mortality was due to the greater technical difficulties that MVR and multiple valve replacement entail. The significantly higher early morality with aortic regurgitation as opposed to aortic stenosis in AVR was not totally unexpected and was probably related to the fact that left ventricular function is better preserved in aortic stenosis. Like other authors, ${ }^{16}$ we found that the nature of the mitral leision (i.e., regurgitation, stenosis, or mixed) had no prognostic significance for early death.

Because a continuous suture technique ${ }^{17}$ was used to implant the vast majority ( $98 \%$ ) of all the valves in this series, we wondered whether this technique could account for the significantly higher rate of periprosthetic leak that we found in the MVR group compared with the AVR group. However, when we looked at the experience of others with various valves, we found that they too had a higher prevalence of periprosthetic leak with mitral compared with aortic valve replacement. For instance, Lindblom with the Björk-Shiley prosthesis (Shiley, Inc.,
Irvine, Calif. $)^{8,9}$ found a much greater (3.5 times) prevalence of periprosthetic leak in the mitral compared with the aortic position despite having used interrupted sutures in both locations. In addition, Keenan and associates ${ }^{17}$ who used an interrupted technique with mitral Medtronic prostheses (Medtronic, Inc., Minneapolis, Minn.) reported a prevalence of periprosthetic leak similar to our own. We concluded, therefore, that no one suture technique could be implicated in causing periprosthetic leak but that there is an impression that the mitral location has a higher prevalence of periprosthetic leak than does the aortic.

Our overall early mortality of $4 \%$ compares favorably with that reported previously for the St. Jude Medical valve $^{3-6,19-24}$ and other mechanical prostheses. ${ }^{7-12,18,25-27}$ Of the 47 early deaths, because of the high rate of postmortem examination $(60 \%)$ and because of the close clinical monitoring especially with echocardiography and fluoroscopic valve screening, we are confident that mechanical failure was not a cause of death. It is worth pointing out that the comparison of mortality between different reports has little value in the absence of a comprehensive clinical profile of the patients.

The results of our analysis encourage us to continue using the St. Jude Medical prosthesis with its proven excellent hemodynamics and absence of structural failure over 13 years. One of the major advantages of this valve is its low profile, which makes it especially useful in children and adults with small ventricles, particularly women. We believe that, by refining our anticoagulation regimen as described previously in this article and by possible further modification such as the introduction of low-dose aspirin therapy ${ }^{28}$ into the regimen, we may reduce further the prevalence of systemic embolism and anticoagulantrelated hemorrhage.

\section{REFERENCES}

1. McGoon DC. The risk of thromboembolism following valvular operations: How does one know? J THORAC CARDIOVASC SURG 1984;88:782-6.

2. Edmunds LH, Clark RE, Cohn LH, Miller DC, Weisel RD. Guidelines for reporting morbidity and mortality after cardiac valvular operations. J THORAC CARDIOVASC SURG 1988;96:351-3.

3. Horstkotte D, Schulte H, Bircks W, Strauer B. Unexpected findings concerning thromboembolic complications and anticoagulation after complete 10 year follow up of patients with St. Jude Medical prostheses. J Heart Valve Dis 1993; 2:291-301.

4. Arom KV, Nicoloff DM, Kersten TE, et al. Ten years' experience with the St. Jude medical valve prosthesis. Ann Thorac Surg 1989;47:831-7.

5. Czer LSC, Chaux A, Matloff JM, et al. Ten-year experi- 
ence with the St. Jude Medical valve for primary valve replacement. J THORAC CARDIOVASC SURG 1990;100:4455.

6. Burckhardt D, Striebel D, Vogt S, et al. Heart valve replacement with St. Jude Medical valve prosthesis: longterm experience in 743 patients in Switzerland. Circulation 1988;78 (Suppl):I18-24.

7. Orszulak TA, Schaff HV, DeSmet J, Danielson GK, Pluth JR, Puga FJ. Late results of valve replacement with the Bjork-Shiley valve (1973 to 1982). J THORAC CARDIOVASC SURG 1993;105:302-12.

8. Lindblom D. Long-term clinical results after aortic valve replacement with the Bjork-Shiley prosthesis. J THORAC Cardiovasc Surg 1988;95:658-67.

9. Lindblom D. Long-term clinical results after mitral valve replacement with the Björk-Shiley prosthesis. J THORAC CARDIOVASC SURG 1988;95:321-33.

10. Antunes MJ, Wessels A, Sadowski RG, et al. Medtronic Hall valve replacement in a third-world population group: a review of the performance of 1000 prostheses. J THORAC CARDiOvasc SuRG 1988;95:980-93.

11. Nitter-Hauge $S$, Abdelnoor M. Ten-year experience with the Medtronic Hall valvular prosthesis: a study of 1104 patients. Circulation 1989;80(Suppl):I43-8.

12. Schoevaerdts JC, Buche M, El Gariani A, et al. Twenty years' experience with the model 6120 Starr-Edwards valve in the mitral position. J THORAC CARDIOvaSC SURG 1987; 94:375-82.

13. Cobanoglu A, Fessler CL, Guvendik L, Grunkemeier G, Starr A. Aortic valve replacement with the Starr-Edward prosthesis: a comparison of the first and second decades of follow-up. Ann Thorac Surg 1988;45:248-52.

14. Lund $\mathrm{O}$, Knudsen MA, Pilegaard HK, Magnussen $\mathrm{K}$, Nielsen TT. Long-term performance of Starr-Edwards silastic ball valves and St. Jude Medical bileaflet valves: a comparative analysis of implantations during 1980-1986 for aortic stenosis. Eur Heart J 1990;11:108-19.

15. Kuntze CEE, Ebels T, Eijgelaar A, Homan Van Der Heide JN. Rates of thromboembolism with three different mechanical heart valve prostheses: randomised study. Lancet 1989;1:514-7.

16. Christakis GT, Weisel RD, David TE, Salerno TA, Ivanov J. Predictors of operative survival after valve replacement. Circulation 1988;78(Suppl):I25-34.
17. Cleland $\mathbf{J}, \mathbf{A}$ universally applicable continuous suture technique for insertion of aortic valve replacement. Ann Thorac Surg 1975;19:719-24.

18. Keenan RJ, Armitage JM, Trento A, et al. Clinical experience with the Medtronic-Hall valve prosthesis. Ann Thorac Surg 1990;50:748-53.

19. Baudet EM, Oca CC, Roques XF, et al. A $5 \frac{1 / 2}{2}$ year experience with the St. Jude Medical cardiac valve prosthesis. J Thorac Cardiovasc Surg 1985;90:137-44.

20. Myers ML, Lawrie GM, Crawford ES, et al. The St. Jude valve prosthesis: analysis of the clinical results in 815 implants and the need for systemic anticoagulation. J Am Coll Cardiol 1989;13:57-62.

21. Kinsley RH, Antunes MJ, Colsen PR. St. Jude Medical valve replacement: an evaluation of valve performance. $J$ Thorac Cardiovasc Surg 1986;92:349-60.

22. Fiore AC, Naunheim KS, D'orazio S, et al. Mitral valve replacement: randomized trial of St. Jude and MedtronicHall prostheses. Ann Thorac Surg 1992;54:68-73.

23. Arom KV, Nicoloff DM, Kersten TE, Northrup WF, Lindsay WG, Emery RW. Ten-year follow-up study of patients who had double valve replacement with the St. Jude Medical prosthesis. J Thorac Cardiovasc Surg 1989;98:1008-16.

24. Armenti F, Stephenson LW, Edmunds LH. Simultaneous implantation of St. Jude Medical aortic and mitral prostheses. J Thorac Cardiovasc Surg 1987;94:733-9.

25. Antunes MJ. Clinical performance of St. Jude and Medtronic-Hall prostheses: a randomized comparative study. Ann Thorac Surg 1990;50:743-7.

26. Aris A, Padro JM, Camara ML, Crexells C, Auge JM, Caralps JM. Clinical and hemodynamic results of cardiac valve replacement with the monostrut Bjork-Shiley prosthesis. J Thorac Cardiovasc Surg 1988;95:42331.

27. Flemma RJ, Mullen DC, Kleinman LH, Werner $\mathbf{P H}$, Anderson AJ, Weirauch E. Survival and "event-free" analysis of 785 patients with Bjork-Shiley spherical-disc valves at 10 to 16 years. Ann Thorac Surg 1988;45:25872.

28. Turpie AGG, Gent M, Laupacis A, Latour Y, Gunstensen J, Basile F, Klimek M, Hirsh J. A comparison of Aspirin with placebo in patients treated with Warfarin after heartvalve replacement. N Engl J Med 1993;524-9. 